\title{
ELECTRON-ION RECOMBINATION OF Fe XII FORMING Fe XI: LABORATORY MEASUREMENTS AND THEORETICAL CALCULATIONS
}

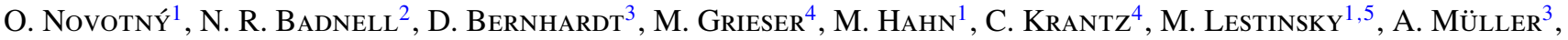 \\ R. RePNOW ${ }^{4}$, S. SCHIPPERS ${ }^{3}$, A. WOLF ${ }^{4}$, AND D. W. SAVIN ${ }^{1}$ \\ ${ }^{1}$ Columbia Astrophysics Laboratory, Columbia University, New York, NY 10027, USA; oldrich.novotny @ mpi-hd.mpg.de \\ ${ }^{2}$ Department of Physics, University of Strathclyde, Glasgow G4 0NG, UK \\ ${ }^{3}$ Institut für Atom- und Molekülphysik, Justus-Liebig-Universität Giessen, D-35392 Giessen, Germany \\ ${ }^{4}$ Max Planck Institute for Nuclear Physics, 69117 Heidelberg, Germany \\ Received 2012 March 26; accepted 2012 April 26; published 2012 June 13
}

\begin{abstract}
We have measured electron-ion recombination for Fe XII forming Fe XI using a merged-beam configuration at the heavy-ion storage ring TSR located at the Max Planck Institute for Nuclear Physics in Heidelberg, Germany. The measured merged-beam recombination rate coefficient (MBRRC) for collision energies from 0 to $1500 \mathrm{eV}$ is presented. This work uses a new method for determining the absolute MBRRC based on a comparison of the ion beam decay rate with and without the electron beam on. For energies below $75 \mathrm{eV}$, the spectrum is dominated by dielectronic recombination (DR) resonances associated with $3 s \rightarrow 3 p$ and $3 p \rightarrow 3 d$ core excitations. At higher energies, we observe contributions from $3 \rightarrow N^{\prime}$ and $2 \rightarrow N^{\prime}$ core excitation DR. We compare our experimental results to state-of-the-art multi-configuration Breit-Pauli (MCBP) calculations and find significant differences, both in resonance energies and strengths. We have extracted the DR contributions from the measured MBRRC data and transformed them into a plasma recombination rate coefficient (PRRC) for temperatures in the range of $10^{3}-10^{7} \mathrm{~K}$. We show that the previously recommended DR data for Fe XII significantly underestimate the PRRC at temperatures relevant for both photoionized plasmas (PPs) and collisionally ionized plasmas (CPs). This is contrasted with our MCBP PRRC results, which agree with the experiment to within 30\% at PP temperatures and even better at CP temperatures. We find this agreement despite the disagreement shown by the detailed comparison between our MCBP and experimental MBRRC results. Last, we present a simple parameterized form of the experimentally derived PRRC for easy use in astrophysical modeling codes.
\end{abstract}

Key words: atomic data - atomic processes - galaxies: active - galaxies: nuclei - plasmas - X-rays: galaxies

\section{INTRODUCTION}

Iron $\mathrm{M}$-shell ions have been identified as the dominant source of the 15-17 $\AA$ absorption feature seen in Chandra and XMM-Newton X-ray observations of warm absorbers in active galactic nuclei (AGNs; e.g., Sako et al. 2001; Holczer et al. 2010). This feature can be used to diagnose the properties of AGNs (Behar et al. 2001). Such a study, however, requires reliable low-temperature dielectronic recombination (DR) rate coefficients for iron M-shell ions, as has been discussed by Netzer (2004), Kraemer et al. (2004), Chakravorty et al. (2008), and Kallman (2010).

A series of experimental and theoretical studies has been performed to meet this need (Gu 2004; Badnell 2006a, 2006b; Altun et al. 2006, 2007; Schmidt et al. 2006, 2008; Lukić et al. 2007; Lestinsky et al. 2009). The measurements in this series are based on a storage ring merged-beam technique utilizing the TSR heavy-ion storage ring located at the Max Planck Institute for Nuclear Physics in Heidelberg, Germany (Habs et al. 1989). A bibliographic compilation of storage ring DR measurements for astrophysically relevant ions has recently been given by Schippers (2009) and an overview of TSR experiments on Fe ions is given by Schippers et al. (2010).

As part of this effort, here we present new experimental results for P-like Fe XII forming S-like Fe XI. Throughout the rest of this paper recombining systems are identified by their initial charge state. The most relevant channels for DR of Fe XII are

\footnotetext{
5 Present address: GSI Helmholtzzentrum für Schwerionenforschung mbH, D-64291 Darmstadt, Germany.
}

$$
\begin{aligned}
& \mathrm{Fe}^{11+}\left(3 s^{2} 3 p^{3}\left[{ }^{4} S_{3 / 2}^{\mathrm{o}}\right]\right)+\mathrm{e}^{-} \\
& \quad \rightarrow\left\{\begin{array}{l}
\mathrm{Fe}^{10+}\left(3 s^{2} 3 p^{3}\left[{ }^{2} D_{3 / 2 ; 5 / 2}^{\mathrm{o}} ;{ }^{2} P^{\mathrm{o}}{ }_{1 / 2 ; 3 / 2}\right] n l\right) \\
\mathrm{Fe}^{10+}\left(3 s 3 p^{4} n l\right) \\
\mathrm{Fe}^{10+}\left(3 s^{2} 3 p^{2} 3 d n l\right) \\
\mathrm{Fe}^{10+}\left(3 s 3 p^{3} 3 d n l\right) .
\end{array}\right.
\end{aligned}
$$

The incident electron is captured into a Rydberg level with a principal quantum number denoted by $n$. DR proceeds via excitation of a core electron with a principal quantum number which we denote by $N$. The energies of the core excitations corresponding to $\Delta N=N^{\prime}-N=0$ DR are listed in Table 1. Fe XII is predicted to form at plasma temperatures of $\log T_{\mathrm{e}}(\mathrm{K}) \sim 4.76-5.48$ in photoionized gas (Kallman 2010) and $\sim 5.87-6.25$ in collisionally ionized gas (Bryans et al. 2006, $2009)$, where $T_{\mathrm{e}}$ is the electron temperature in Kelvin.

The remainder of this paper is organized as follows: Section 2 gives a brief summary of the theoretical calculations. Section 3 describes the experimental setup used here. Our experimental results for the merged-beam recombination rate coefficient (MBRRC) are presented and compared to theory in Section 4. Section 5 reports our experimentally derived DR plasma recombination rate coefficient (PRRC), a comparison with theory, and a simple fitting formula for plasma modeling. Last, a summary is given in Section 6.

\section{THEORY}

The partial, energy-averaged, DR cross-section $\bar{\sigma}_{f i}^{z}$ from an initial state $i$ of an ion $X^{+z}$ into a resolved final state $f$ of an 
Table 1

Energy Levels of Fe XII Relative to the $3 s^{2} 3 p^{3}\left[{ }^{4} S_{3 / 2}^{o}\right]$ Ground Level (Ralchenko et al. 2011) for Excitations within the M-shell $(\Delta N=0)$

\begin{tabular}{lc}
\hline \hline Level & Energy \\
& $(\mathrm{eV})$ \\
\hline $3 s^{2} 3 p^{3}\left[{ }^{2} D_{3 / 2}^{o}\right]$ & 5.1535 \\
$3 s^{2} 3 p^{3}\left[{ }^{2} D_{5 / 2}^{o}\right]$ & 5.7126 \\
$3 s^{2} 3 p^{3}\left[{ }^{2} P_{1 / 2}^{o}\right]$ & 9.1883 \\
$3 s^{2} 3 p^{3}\left[{ }^{2} P_{5 / 2}^{o}\right]$ & 9.9826 \\
$3 s 3 p^{4}\left[{ }^{4} P_{5 / 2}\right]$ & 34.0179 \\
$3 s 3 p^{4}\left[{ }^{4} P_{3 / 2}\right]$ & 35.2121 \\
$3 s 3 p^{4}\left[{ }^{4} P_{1 / 2}\right]$ & 35.7455 \\
$3 s 3 p^{4}\left[{ }^{2} D_{3 / 2}\right]$ & 42.1571 \\
$3 s 3 p^{4}\left[{ }^{2} D_{5 / 2}\right]$ & 42.3658 \\
$3 s 3 p^{4}\left[{ }^{2} P_{3 / 2}\right]$ & 48.3174 \\
$3 s 3 p^{4}\left[{ }^{2} S_{1 / 2}\right]$ & 48.8646 \\
$3 s^{2} 3 p^{2}\left({ }^{1} D\right) 3 d\left[{ }^{2} P_{3 / 2}\right]$ & 62.2153 \\
$3 s^{2} 3 p^{2}\left({ }^{3} P\right) 3 d\left[{ }^{4} P_{5 / 2}\right]$ & 63.5431 \\
$3 s 3 p^{4}\left[{ }^{2} P_{1 / 2}\right]$ & 63.7093 \\
$3 s^{2} 3 p^{2}\left({ }^{3} P\right) 3 d\left[{ }^{4} P_{3 / 2}\right]$ & 64.0676 \\
$3 s^{2} 3 p^{2}\left({ }^{3} P\right) 3 d\left[{ }^{4} P_{1 / 2}\right]$ & 64.4433 \\
$3 s^{2} 3 p^{2}\left({ }^{1} S\right) 3 d\left[{ }^{2} D_{3 / 2}\right]$ & 65.2306 \\
$3 s^{2} 3 p^{2}\left({ }^{1} S\right) 3 d\left[{ }^{2} D_{5 / 2}\right]$ & 66.7085 \\
$3 s^{2} 3 p^{2}\left({ }^{1} D\right) 3 d\left[{ }^{2} D_{3 / 2}\right]$ & 68.6910 \\
$3 s^{2} 3 p^{2}\left({ }^{1} D\right) 3 d\left[{ }^{2} D_{5 / 2}\right]$ & 68.7629 \\
$3 s^{2} 3 p^{2}\left({ }^{1} D\right) 3 d\left[{ }^{2} P_{1 / 2}\right]$ & 70.5396 \\
$3 s^{2} 3 p^{2}\left({ }^{3} P\right) 3 d\left[{ }^{2} F_{5 / 2}\right]$ & 71.5066 \\
$3 s^{2} 3 p^{2}\left({ }^{3} P\right) 3 d\left[{ }^{2} P_{3 / 2}\right]$ & 71.6306 \\
$3 s^{2} 3 p^{2}\left({ }^{1} D\right) 3 d\left[{ }^{2} S_{1 / 2}\right]$ & 71.8650 \\
$3 s^{2} 3 p^{2}\left({ }^{3} P\right) 3 d\left[{ }^{2} F_{7 / 2}\right]$ & 72.0571 \\
$3 s^{2} 3 p^{2}\left({ }^{3} P\right) 3 d\left[{ }^{2} D_{5 / 2}\right]$ & 74.8778 \\
$3 s^{2} 3 p^{2}\left({ }^{3} P\right) 3 d\left[{ }^{2} D_{3 / 2}\right]$ & 75.0699 \\
\hline & \\
\hline & \\
&
\end{tabular}

ion $X^{+z-1}$ is given in the isolated resonance approximation by (Burgess 1964; Badnell 2006a)

$$
\bar{\sigma}_{f i}^{z}\left(E_{\mathrm{c}}\right)=\frac{2 \tau_{0}\left(\pi a_{0} I_{\mathrm{H}}\right)^{2}}{E_{\mathrm{c}} \Delta E} \sum_{j} \frac{\omega_{j}}{\omega_{i}} \frac{\sum_{l} A_{j \rightarrow i, E_{\mathrm{c}} l}^{\mathrm{a}} A_{j \rightarrow f}^{\mathrm{r}}}{\sum_{h} A_{j \rightarrow h}^{\mathrm{r}}+\sum_{m, l} A_{j \rightarrow m, E_{\mathrm{c}}}^{\mathrm{a}}} .
$$

Here, $\omega_{j}$ is the statistical weight of the doubly excited resonance state $j$ in the recombined $+z-1$ ion, $\omega_{i}$ is the statistical weight of the initial state of the initial $+z$ ion, and the autoionization $\left(A^{\mathrm{a}}\right)$ and radiative $\left(A^{\mathrm{r}}\right)$ rates are in inverse seconds. The indices $h$ and $m$ are for states in the $+z-1$ and $+z$ ions, respectively. $E_{\mathrm{c}}$ is the energy of the incoming continuum electron (with orbital angular momentum $l$ ) which is fixed by the position of the resonances, $\Delta E$ is an arbitrary bin width, $I_{\mathrm{H}}$ is the ionization potential energy of the hydrogen atom, $\tau_{0}$ is the atomic unit of time, and $a_{0}$ is the Bohr radius.

We use the general atomic collision code AUTOSTRUCTURE (Badnell 1986, 2006a, 2011) to calculate the constituents of Equation (2). The calculations for $\Delta N=0$ core excitations were carried out in intermediate coupling using a configuration interaction expansion for the $\mathrm{Fe}^{11+} 15$-electron target involving a Ne-like core and valence configurations of $3 s^{2} 3 p^{3}, 3 s 3 p^{4}$, $3 s^{2} 3 p^{2} 3 d, 3 p^{5}, 3 s 3 p^{3} 3 d, 3 s^{2} 3 p 3 d^{2}, 3 p^{4} 3 d$, and $3 s 3 p^{2} 3 d^{2}$ to which continuum and Rydberg electron orbitals were coupled. The 16-electron configurations, formed by adding a $3 s$, $3 p$, or $3 d$ orbital to the 15 -electron configurations, were included to describe outer electron radiative transitions into the core. Radiative transitions from higher $n$ levels were described hydrogenically. The merged-beam experiment does not resolve the final state and so all of the results that we present are for the total recombination cross-section, i.e., summed over all $f$ that are stable against autoionization and are not field ionized in TSR before they are detected. These energy-averaged cross-sections can be convolved with the experimental energy distribution for comparison with the measurements. They can also be convolved with a Maxwellian distribution for modeling use and summed over all possible stable final states to generate a total PRRC. For $\Delta N=0 \mathrm{DR}$, the sum over the Rydberg $n l$ states extended to $n=1000$ and $l=11$ for the total Maxwellian rate coefficients while for comparison with experiment the relevant survival probabilities were folded into the sum over the final states (e.g., Schippers et al. 2001).

The contributions from $\Delta N>0$ core excitations were also calculated by AUTOSTRUCTURE but using a configurationaveraged approximation (Pindzola et al. 1986). This approximation is only suited for $\Delta N>0$ Maxwellian rate coefficients since it only resolves resonance positions and channels by configuration only. The omission of configuration mixing is not a severe one for the total PRRC given that mixing conserves the overall amount of resonance strengths and, at the energies relevant here, causes only small fractional errors in the resonance energies (Badnell et al. 2011). We include both $N=2 \rightarrow 3$ and $N=3 \rightarrow 4$ core excitations. The sum over the captured electron $n l$ Rydberg states extended to $n=100$ and $l=6$ for these total Maxwellian rate coefficients. No difference is seen between the calculations with and without the field ionization effects included.

\section{EXPERIMENTAL SETUP}

\subsection{General}

Measurements were performed using the heavy-ion storage ring TSR. Details on the various aspects of the merged-beam technique as used at TSR have been described at length by Kilgus et al. (1992), Lampert et al. (1996), Pastuszka et al. (1996), Schippers et al. (2001), Wolf et al. (2006), Lestinsky et al. (2008), Schmidt et al. (2008), and Lestinsky et al. (2009). Here, we discuss only those aspects particular to the present work.

A $150 \mathrm{MeV}$ beam of ${ }^{56} \mathrm{Fe}^{11+}$ was generated by first passing ${ }^{56} \mathrm{Fe}^{-}$ions through a carbon foil to strip and produce the desired charge state and then further accelerating them. After chargeto-mass selection, the $\mathrm{Fe}^{11+}$ beam was injected into the storage ring. Ions were accumulated by multi-turn injection and "e-cool stacking" (Grieser et al. 1991). Typical stored ion currents were $\sim 1-2 \mu$ A during data acquisition with storage times of $\approx 10 \mathrm{~s}$.

Ions produced by foil stripping can be highly excited (Martinson \& Gaupp 1974). Here, we stored the ions for $\sim 1.5-2.0 \mathrm{~s}$ before beginning data acquisition. We used a numerical model of the radiative decay process to determine the level populations in the ion beam after this initial storage time. The model considered excited states up to the $3 s^{2} 3 p^{2} 4 d^{2} S_{1 / 2}$ level and included all 66 transitions, involving 31 levels, for which radiative rates are given in the ASD/NIST database (Ralchenko et al. 2011). These data, in turn, come from Fawcett et al. (1972), Huang (1984), and Shirai et al. (1990). The initial relative populations of the excited states were modeled using a Boltzmann distribution with a temperature of $k_{\mathrm{B}} T=750 \mathrm{eV}$. This corresponds to the approximate collision energy of the foil electrons as the $\mathrm{Fe}^{-}$ions passed through the carbon foil. After $1.5 \mathrm{~s}$ of storage, over $98.5 \%$ of the ion beam is expected to be in the ground state. A factor of 10 increase or decrease in the effective temperature has an insignificant effect on this estimate. The most critical lifetime determining the final population is that of 
the $3 s^{2} 3 p^{3}\left[{ }^{2} D_{5 / 2}^{o}\right] \rightarrow 3 s^{2} 3 p^{3}\left[{ }^{4} S_{3 / 2}^{o}\right]$ radiative transition to the ground state. The Einstein coefficient listed in ASD/NIST database at $1.84 \mathrm{~s}^{-1}$ is smaller compared to the more recent experimental value of $3.26 \mathrm{~s}^{-1}$ reported by Träbert et al. (2002). Using the latter result, the predicted ground-state population after $1.5 \mathrm{~s}$ of storage is $99.0 \%$. For both lifetimes, the ground-state population averaged over the entire $\sim 20$ s storage time is greater than $99.9 \%$.

TSR is equipped with two different electron beam devices located in separate sections of the ring. Each electron beam can be merged to co-propagate with the stored ions. One of the devices is called the Cooler (Steck et al. 1990), and the other is called the Target (Sprenger et al. 2004). Either or both of the electron beams can serve to reduce the energy spread of the ions, i.e., to cool the ions. Electron cooling (Poth 1990) results in a narrow ion beam diameter $(<1 \mathrm{~mm})$ with a low energy spread. Additionally, either one of the electron beams can be used as an interaction medium while the other continues to cool the ion beam. Electron-ion collisions can then be investigated by varying the energy of one of the electron beams.

The electron beam energy spread is described by a flattened Maxwellian distribution characterized by the longitudinal and transverse temperatures $T_{\|}$and $T_{\perp}$ (Kilgus et al. 1992). At a collision energy of $\hat{E}$, the corresponding centerof-mass collision energy resolution $\Delta \hat{E}$ is approximately given by $\Delta \hat{E}=\left[\left(\ln (2) k_{\mathrm{B}} T_{\perp}\right)^{2}+16 \ln (2) \hat{E} k_{\mathrm{B}} T_{\|}\right]^{1 / 2}$ (Müller 1999). The Cooler uses a thermionic emission cathode. Typical electron beam temperatures are $k_{\mathrm{B}} T_{\|}^{\mathrm{c}} \approx 180 \mu \mathrm{eV}$ and $k_{\mathrm{B}} T_{\perp}^{\mathrm{c}} \approx 13.5 \mathrm{meV}$ (Lestinsky et al. 2008). The Target uses a photocathode (Pastuszka et al. 2000; Orlov et al. 2004). From this we produce a beam with significantly lower temperatures of $k_{\mathrm{B}} T_{\|}^{\mathrm{t}} \approx 25 \mu \mathrm{eV}$ and $k_{\mathrm{B}} T_{\perp}^{\mathrm{t}} \lesssim 1.5 \mathrm{meV}$ (Lestinsky et al. 2008). The complexity of the $\mathrm{Fe}^{11+}$ DR spectrum prevented direct determination of the Cooler and Target temperatures from the measured spectrum. Hence, for the results presented here we used values from a similar experiment (Lestinsky et al. 2008).

The products of charge-changing reactions are deflected from the parent ion beam by the first dipole magnet downstream of each electron beam device and are directed onto a detector. Scintillator detectors for measuring recombination are located after both the Cooler and the Target (Miersch et al. 1996; Wissler 2002; Lestinsky 2007). To measure electron impact ionization (EII), we used a converter plate coupled with a channel electron multiplier (Rinn et al. 1982; Linkemann et al. 1995) located after the Cooler. The recombination and ionization signals were used to determine the absolute recombination rate coefficient from the ion beam lifetime as described below.

The efficiency of each detector, in the absence of dead time effects, is essentially $100 \%$. The dead time for each detector was estimated from the maximal width of the electronic pulses. This was shorter than $100 \mathrm{~ns}$ in all cases. As the count rates never exceeded $300 \mathrm{kHz}$, the corresponding dead-time corrected detector efficiency did not decrease below $97 \%$.

Field ionization of the recombined ions in the dipole magnet can ionize electrons captured into Rydberg levels with $n \geqslant n_{\text {cut }}$. A semiclassical calculation yields $n_{\text {cut }}=42$. However, during the travel time from the interaction region to the dipole, some of the initially high Rydberg states can radiatively decay below $n_{\text {cut }}$ and avoid ionization in the magnet. Schippers et al. (2001) have derived a model to calculate $n l$-specific detection probabilities taking into account the field ionization and radiative de-excitation processes. The average Rydberg state cutoff resulting from this model is $n_{\text {cut }} \approx 54$. For comparison of our results to the theory, we use the detection probabilities provided by this model.

\subsection{Determination of the Relative MBRRC Spectra}

Normally the absolute MBRRC is derived from the measured recombination counts using an appropriate normalization to the electron density and ion current (e.g., Schmidt et al. 2008). The electron density can be readily measured accurately (e.g., Lestinsky et al. 2009). However, here the average stored ion current of $\sim 1-2 \mu \mathrm{A}$ in the present experiment was too low to be directly measured using the DC current transformer installed in the ring. Instead, a relative MBRRC was determined by normalizing the signal count rate to a proxy for the ion current. For this we used the relative intensity of the ion beam as recorded by a beam profile monitor (Hochadel et al. 1994). To derive the absolute calibration of the MBRRC spectrum, we use the approach described in Section 3.3.

Data were collected for electron beam laboratory energies from $\sim 1450$ to $\sim 6000 \mathrm{eV}$. These translate to center-of-mass collision energies $0 \mathrm{eV} \leqslant \hat{E} \leqslant 1500 \mathrm{eV}$, where $\hat{E}=0$ corresponds to matched electron and ion beam velocities. The full range was covered using the Cooler to collect data with continuous cooling by the Target. The high electron beam density of $n_{\mathrm{e}} \approx 2.7 \times 10^{7} \mathrm{~cm}^{-3}$ in the Cooler allowed for shorter data acquisition times than that of the Target which had a density of $n_{\mathrm{e}} \approx 1.1 \times 10^{6} \mathrm{~cm}^{-3}$. The roles were reversed to cover the low energy range $0 \mathrm{eV} \leqslant \hat{E} \leqslant 0.2 \mathrm{eV}$ at the higher energy resolution offered by the Target. As discussed in Section 4, these data aided in the extraction of the low energy DR resonance strengths needed to generate a reliable low-temperature PRRC.

Each data run lasted $\sim 1-2 \mathrm{hr}$, during which time we continuously repeated the measurement cycle of ion injection, cooling, and data acquisition. During data collection, the electron beam energy was stepped between a variable measurement energy and a fixed reference energy, creating $\sim 350$ measurement pairs of typically $\lesssim 20$ s total duration. The measurement energy was changed after each reference step. For each data run, the measurement energy range in the laboratory frame spanned over $\sim 140-1000 \mathrm{eV}$. This is much smaller than the total laboratory energy range studied. The reason for splitting to smaller energy ranges is related to the required settling time of the power supplies when switching the electron beam laboratory energy to measurement or reference. In order to keep this time short (15 $\mathrm{ms}$ in our case), the reference laboratory energy in each run was kept close to the measurement laboratory energy range. As a result, several data runs were needed to cover the entire laboratory energy range measured. After the $15 \mathrm{~ms}$ settling time, the subsequent dwell time at each measurement or reference energy step was $10-25 \mathrm{~ms}$.

The signal at reference consists of background due to electron capture from residual gas, radiative recombination (RR), and potentially also DR. At high energies, DR is negligible and so when we subtracted the reference from the measurement it was only necessary to re-add the small theoretical RR contribution, thereby insuring that only the appropriate background was subtracted. In the low energy runs, however, the DR contribution at the reference energy became non-negligible. We corrected for this by comparing a lower energy run to an overlapping higher energy run and shifting the offset in the former to match the data in the latter. 

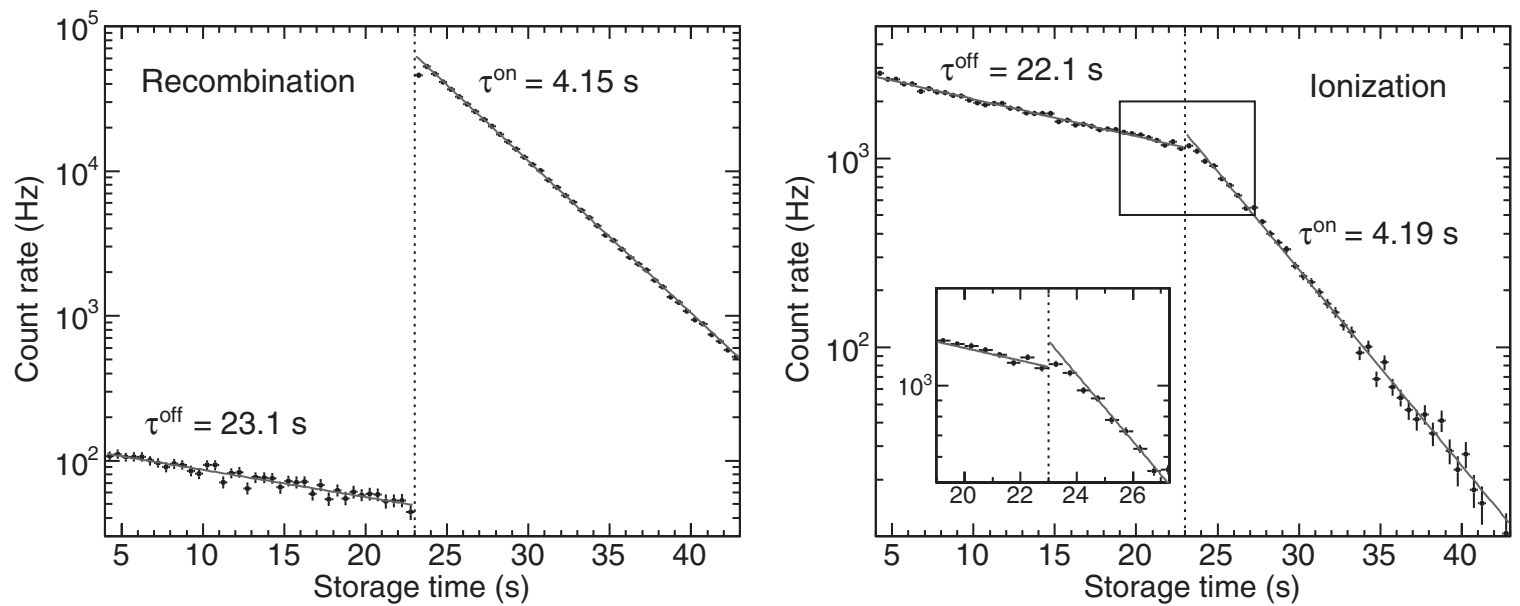

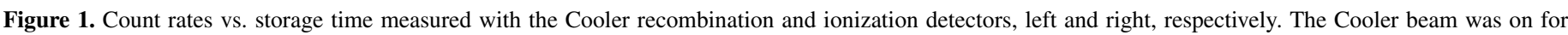

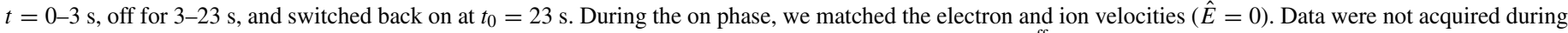

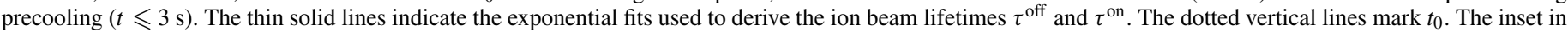

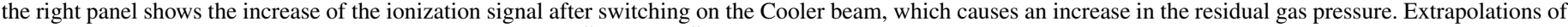
the solid lines to $t_{0}$ were used to determine $R_{\mathrm{c}}^{1 \text {,on }}, R_{\mathrm{c}}^{2 \text {,on }}, R_{\mathrm{c}}^{1 \text {, off }}$, and $R_{\mathrm{c}}^{2 \text {,off }}$. See Appendix A for details.

\subsection{Absolute Scaling of MBRRC Spectra}

We put our relative MBRRC results on an absolute scale using measurements of the ion beam lifetime with the Cooler electron beam first off and then on. A similar normalization method has been used for molecular ion studies (Pedersen et al. 2005). Our approach builds upon and extends their work.

With the Cooler off, the ion beam decays exponentially due to collisions with the residual gas along the entire TSR circumference. The measured storage lifetime $\tau^{\text {off }}$ is inversely proportional to the loss rate $\lambda_{\text {res }}^{\text {off }}$ as

$$
\frac{1}{\tau^{\text {off }}}=\lambda_{\text {res }}^{\text {off }} .
$$

With the Cooler on at a fixed energy $\hat{E}$, the measured storage lifetime is now due to both collisions with the residual gas and also to RR and DR in the Cooler, giving

$$
\frac{1}{\tau^{\text {on }}}=\lambda_{\text {res }}^{\text {on }}+\hat{\alpha} n_{e} \eta
$$

Here, $\lambda_{\text {res }}^{\text {on }}$ is the residual gas collisional loss rate with the Cooler on, $\hat{\alpha}$ is the $\mathrm{RR}+\mathrm{DR}$ MBRRC at $\hat{E}, n_{e}$ is the Cooler electron density, and $\eta=L / C$ is the overlap length $L$ of the ion and electron beams normalized by the total storage ring circumference $C$. As a first approximation, we set $\lambda_{\text {res }}^{\text {off }}=\lambda_{\text {res }}^{\text {on }}$ and solve Equations (3) and (4) to yield an absolute MBRRC

$$
\hat{\alpha}(\hat{E})=\frac{1}{n_{e} \eta}\left(\frac{1}{\tau^{\text {on }}}-\frac{1}{\tau^{\text {off }}}\right) .
$$

A more thorough derivation, accounting for the slight differences between $\lambda_{\text {res }}^{\text {off }}$ and $\lambda_{\text {res }}^{\text {on }}$ due to changes in the pressure of residual gas, is given in Appendix A. Here, this difference results in less than a $5 \%$ change in $\hat{\alpha}(\hat{E})$. The relative MBRRC results of Section 3.2 can then be put on an absolute scale by adjusting the data so that the value at $\hat{E}$ matches the absolute rate derived using this lifetime method.

Figure 1 shows an example of data collected using this method for matched ion and electron beam velocities $(\hat{E}=0 \mathrm{eV})$. For these results, the ions were cooled for $3 \mathrm{~s}$ after injection and then the Cooler beam was switched off. Some time later the Cooler beam was switched back on. The relative beam intensity for each phase was monitored by detecting products from ion collisions resulting in electron capture and ionization. We determined the beam lifetime using the decaying signal strength on both the recombination and ionization detectors. For the results presented here the Target electron beam was on continuously.

For the present work, the measured beam lifetime decreased dramatically when the Cooler was turned on due to the extraordinary high rate coefficient of $\mathrm{DR}+\mathrm{RR}$ at $\hat{E}=0$, which dominates over collisions with the residual gas at the given electron density. This can be seen in both the recombination and ionization detector count rates shown in Figure 1. The Cooler energy is below the Fe XII threshold for EII $(\sim 330 \mathrm{eV}$; Ralchenko et al. 2011) and so the signal on the ionization detector originated exclusively from electron stripping in ion collisions with residual gas. Thus, we attribute the small increase seen in the ionization product count rate when the Cooler is turned on as being due to a corresponding increase in the residual gas pressure. This pressure change is accounted for in our analysis as is described in Appendix A and has less than a 5\% effect on our results. We also found that, to within the statistical errors, the absolute scaling method used here gave the same results independent of when the Cooler was switched on or whether the Target was on or off.

We use the absolute MBRRC results to scale our relative MBRRC data. These are then corrected for the effects due to the merging and demerging of the electrons with the ions (Lampert et al. 1996). This correction largely removes errors due to the uncertainty in the exact electron-ion beams overlap length.

\subsection{Uncertainties}

The $1 \sigma$ statistical error in our rate coefficient data is about $1 \%$ for collision energies below $1 \mathrm{meV}$. As the energy increases, the recombination rate, and hence the signal rate, decreases. This leads to an increase in the statistical error with increasing energy. It remains below $5 \%$ for energies up to $66 \mathrm{eV}$ and $\sim 7 \%$ for $66-1500 \mathrm{eV}$. 
We treat the various systematic errors in our measurement as uncorrelated and add them in quadrature. The resulting $1 \sigma$ systematic error is estimated to be $12 \%, 13 \%$, and $40 \%$ for collision energies of $0 \mathrm{eV}, 66 \mathrm{eV}$, and above $66 \mathrm{eV}$, respectively. Here, we briefly review the sources of the total systematic uncertainty. Further details about systematic errors can be found in the TSR references cited in Section 3.1.

The largest source of systematic error below $66 \mathrm{eV}$ is due to the electron density determination. The unusually high $10 \%$ error for the data here resulted from the accidental use of a degraded photocathode for the absolute MBRRC measurement. The reproducibility of the absolute results and the extraction of ion beam lifetimes for the determination of the absolute MBRRC at $0 \mathrm{eV}$ result in an additional $5 \%$ uncertainty.

The data have been stitched together going from high to low energies to correct for the changing reference energy. As the data have been normalized at $\hat{E}=0$, this stitching results in a $5 \%$ error at $66 \mathrm{eV}$ and up to a $35 \%$ error at higher energies. The large increase in this error above $66 \mathrm{eV}$ is due to the $\sim 100$ times decrease in the magnitude of the MBRRC. Other remaining sources of error include the corrections for the merging and demerging of the beams (1\%; Lampert et al. 1996) and the dead-time counting efficiencies of detectors.

\section{MERGED-BEAM RECOMBINATION RATE COEFFICIENT}

The measured MBRRC data are displayed in Figures 2 and 3 for the energy ranges $0-80 \mathrm{eV}$ and $65-1500 \mathrm{eV}$, respectively. These data were acquired using the Cooler as the probe beam and the Target for cooling. Also shown are our AUTOSTRUCTURE multi-configuration Breit-Pauli (MCBP) results, with and without the experimental field ionization effects. The theoretical cross-section has been multiplied by the collision velocity and convolved with the Cooler energy spread to generate a theoretical MBRRC.

The Fe XII resonance spectrum for this system with a halfopen $p$-shell is very rich and challenging to disentangle. In general, the features are broad and unresolved making individual assignments essentially impossible. DR via $3 s^{2} 3 p^{3}$ intraconfiguration core excitations is expected for energies below $\approx 10 \mathrm{eV}$. The bulk of these contribute significantly only below $\approx 5 \mathrm{eV}$ as can be seen by the step-like drop in the MBRRC at this energy. Moving up in energy, most of the $3 s 3 p^{4}$ core excitations are expected to occur below $\approx 35 \mathrm{eV}$. At energies of $35-75 \mathrm{eV}$, the features become more regular. These are due largely to $3 s^{2} 3 p^{2} 3 d$ core excitations and the resonances can be more easily assigned. For clarity, we have labeled only those resonances which are due to the strongest $3 s 3 p^{4}$ and $3 s^{2} 3 p^{2} 3 d$ series. Filling in the many other resonance series would make the figures too cluttered for meaningful inspection.

Given the complexity of the spectra, for comparison with theory we have followed Lestinsky et al. (2009) and calculated

$$
\kappa=\frac{\int \alpha_{\text {theo }}^{\mathrm{DR}} d E}{\int \alpha_{\exp }^{\mathrm{DR}} d E}
$$

for sequential energy ranges. The lowest energy considered is $13.5 \mathrm{meV}$ to avoid the well-known effects of enhanced RR near $\hat{E}=0$ (Gwinner et al. 2000; Wolf \& Gwinner 2003; Hörndl et al. 2006). In the denominator of Equation (6) we take $\alpha_{\exp }^{\mathrm{DR}}=\alpha_{\text {exp }}-\alpha_{\text {theo }}^{\mathrm{RR}}$. We have calculated $\alpha_{\text {theo }}^{\mathrm{RR}}$ using both the hydrogenic Bethe-Salpeter method (Hoffknecht et al. 2001)
Table 2

Integrated DR Rate Coefficients for Fe XII

\begin{tabular}{lccc}
\hline \hline Energy Range & $\int \alpha_{\text {theo }}^{\mathrm{DR}} d E$ & $\int \alpha_{\exp }^{\mathrm{DR}} d E$ & $\kappa=\frac{\int \alpha_{\text {theo }}^{\mathrm{DR}} d E}{\int \alpha_{\exp }^{\mathrm{DR}} d E}$ \\
$(\mathrm{eV})$ & $\left(10^{-9} \mathrm{~cm}^{3} \mathrm{~s}^{-1} \mathrm{eV}\right)$ & \\
\hline $0.0135-0.45$ & 1.86 & $5.29(2)$ & $0.352(6)$ \\
$0.45-5.5$ & 10.84 & $14.22(2)$ & $0.762(14)$ \\
$5.5-15.0$ & 3.41 & $4.61(1)$ & $0.741(10)$ \\
$15.0-24.5$ & 2.81 & $3.70(2)$ & $0.758(12)$ \\
$24.5-36.0$ & 4.20 & $5.11(1)$ & $0.821(4)$ \\
$36.0-42.0$ & 1.20 & $1.89(1)$ & $0.633(3)$ \\
$42.0-46.0$ & 0.98 & $1.57(1)$ & $0.624(2)$ \\
$46.0-53.0$ & 2.32 & $2.59(1)$ & $0.893(5)$ \\
$53.0-59.0$ & 3.62 & $2.58(1)$ & $1.400(6)$ \\
$59.0-66.0$ & 14.04 & $8.30(1)$ & $1.693(12)$ \\
$66.0-75.0$ & 0.21 & $0.44(1)$ & $0.468(3)$ \\
$75.0-217.0$ & 3.51 & $5.16(3)$ & $0.680(20)$ \\
$330.0-885.0$ & 1.89 & $6.7(2)$ & $0.282(51)$ \\
\hline
\end{tabular}

Note. Here, the values in brackets give the $1 \sigma$ statistical errors for the last digit(s) shown.

and a hydrogenic quantum mechanical dipole approximation for low $n$ and a semiclassical approach with Stobbe corrections for high $n$ (Stobbe 1930). The difference in $\alpha_{\text {theo }}^{\mathrm{RR}}$ between the two methods is insignificant.

We find mixed agreement between theory and experiment. Results for $\kappa$ are given in Table 2. If the difference were solely due to the estimated $1 \sigma$ experimental systematic error, we would expect this ratio to range between $0.88-1.15$ and $0.71-1.67$ for collision energies below and above $66 \mathrm{eV}$, respectively. In the energy range $0.0135-0.45 \mathrm{eV}, \kappa$ is nearly one-third. This is most likely due to incorrectly predicted resonance energies resulting from the well-known difficulty of calculating DR resonance positions at low energies (cf. Schippers 2009 and Schippers et al. 2010, and references therein). In the various energy ranges between $0.45 \mathrm{eV}$ and $46.0 \mathrm{eV}$, theory is smaller by more than the $1 \sigma$ experimental systematic uncertainty. Reasonable agreement is found in the range 46-53 eV. However in the range $53-59 \mathrm{eV}$, theory is 1.4 times greater than experiment. This apparent systematic overestimate of the integrated theoretical resonance strength occurs for $\Delta N=0 \mathrm{DR}$ where the radiative stabilization is primarily by the core electron, and the Rydberg electron occupies $n \gtrsim 10$. Similar discrepancies have been seen in previous work (Lestinsky et al. 2009) and are discussed in more detail by Lestinsky et al. (2012). The range 59-66 eV includes six Rydberg series, mostly with a $3 s^{2} 3 p^{2} 3 d$ configuration, and the large $\kappa$ might be partly explained by uncertainties in the model for the experimental field ionization effect. Last, the range $66-75 \mathrm{eV}$ covers 10 Rydberg series limits associated with the $3 s^{2} 3 p^{2} 3 d$ configuration. The cause of the low $\kappa$ here is unclear.

The MBRRC at energies above $75 \mathrm{eV}$ is dominated by $\Delta N>0$ DR. Here, the MBRRC is $\sim 100$ times weaker than for $\Delta N=0$ at lower energies. Not surprisingly, our configurationaveraged calculations do a poor job of reproducing the observed resonance structure. Between $\sim 75$ and $330 \mathrm{eV}$ the spectrum is expected to be dominated by DR via $3 \rightarrow N^{\prime}$ core excitations where $N^{\prime} \geqslant 4$. The resonances between $\sim 75$ and $217 \mathrm{eV}$ we attribute to $3 \rightarrow 4$ excitations and the region between $\sim 217 \mathrm{eV}$ and the ionization limit of excitations into $N^{\prime} \geqslant 5$. In this first range, we find $\kappa=0.68$. We cannot determine $\kappa$ for the $217-330 \mathrm{eV}$ range as the $N^{\prime} \geqslant 5$ resonances were not included in the theoretical model. The small decrease in the DR signal at $\sim 330 \mathrm{eV}$ corresponds to the $N=3$ ionization threshold 

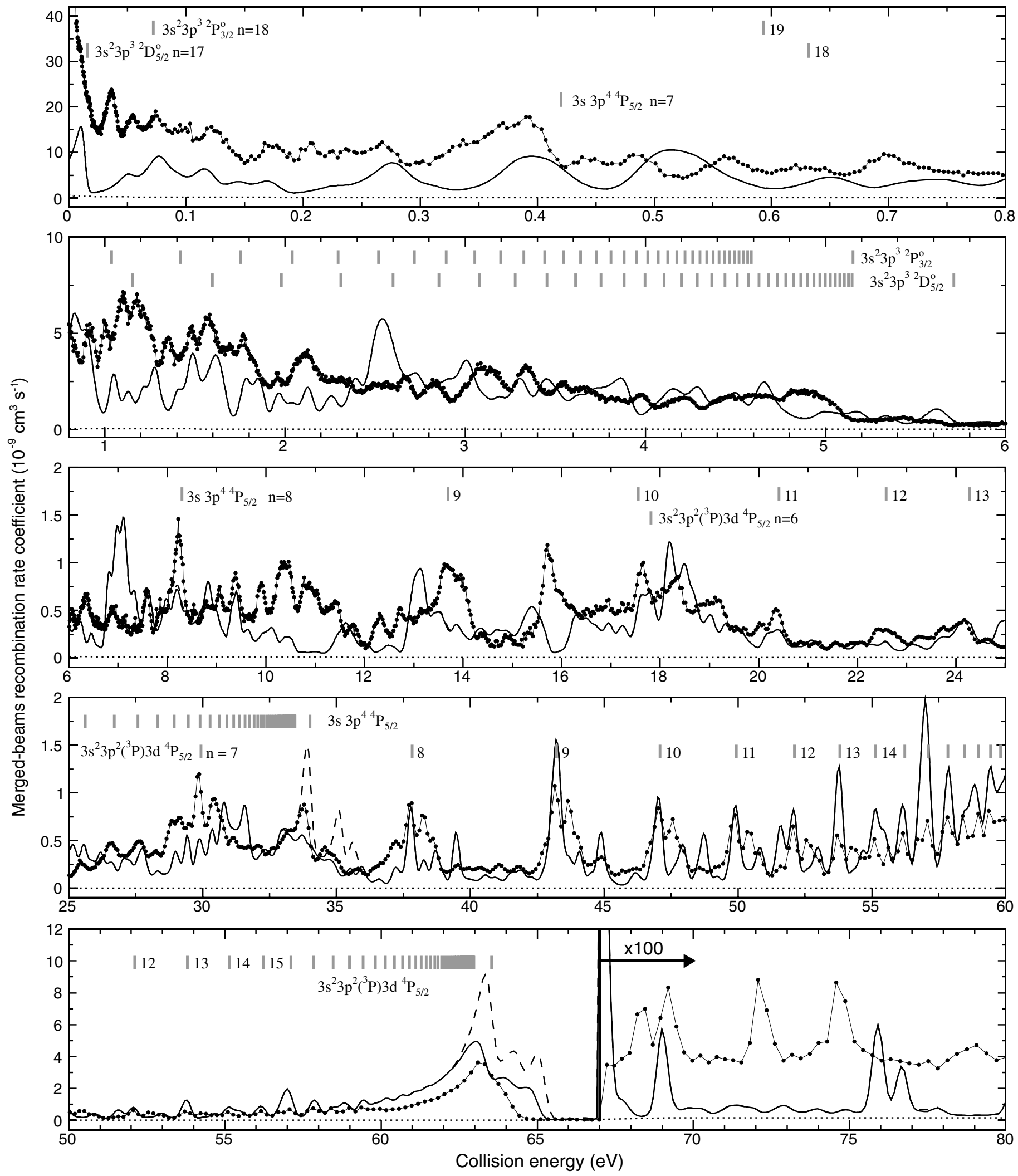

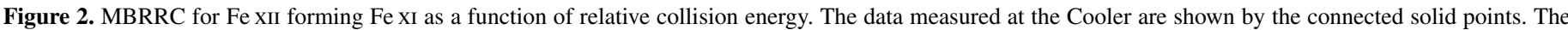

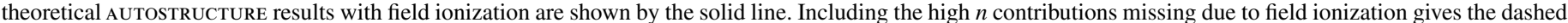

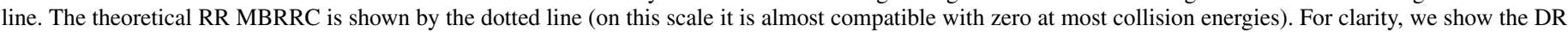

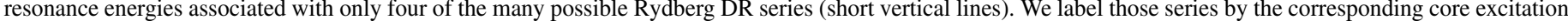
configuration. In each series, the highest energy vertical mark corresponds to the series limit and the penultimate mark to the approximate field ionization cutoff. 


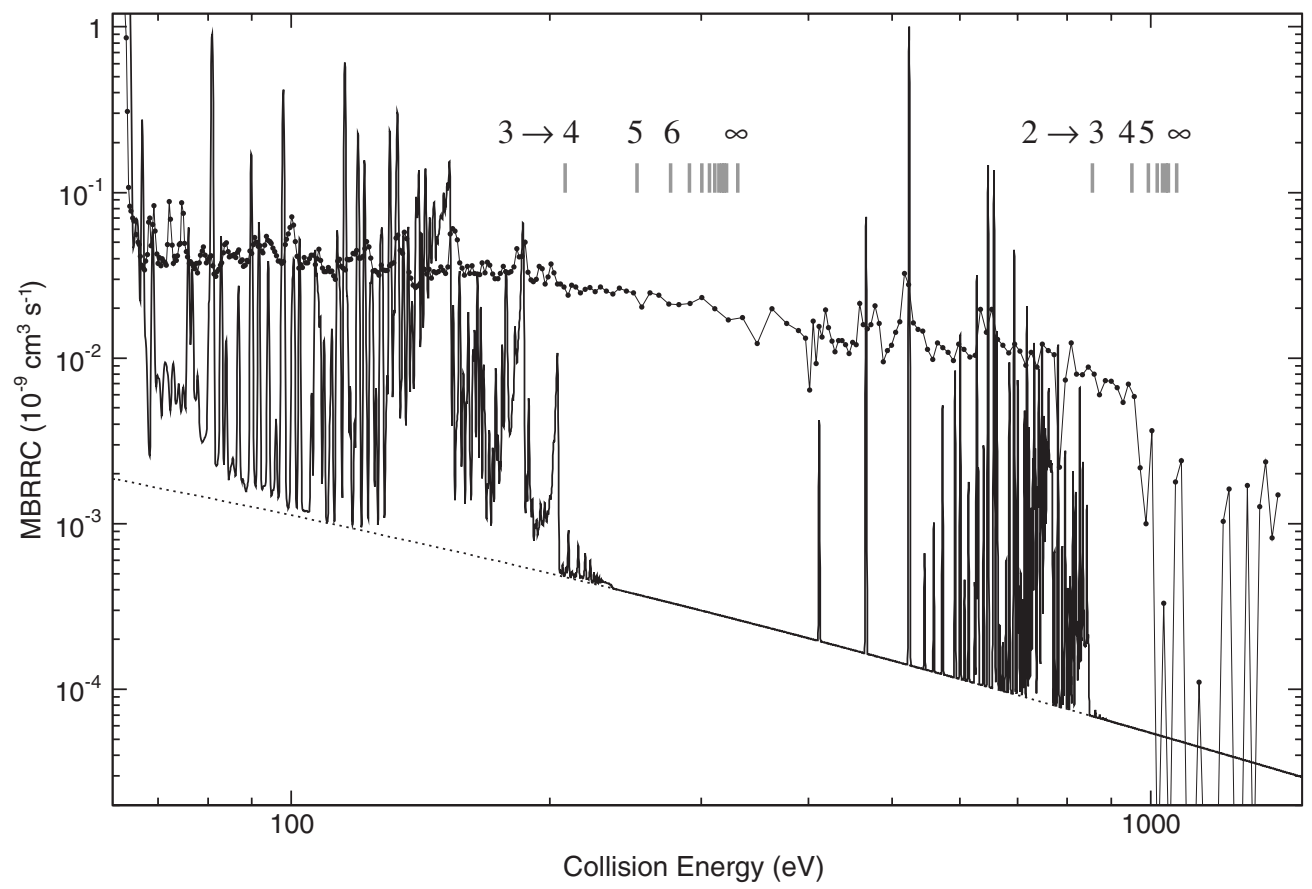

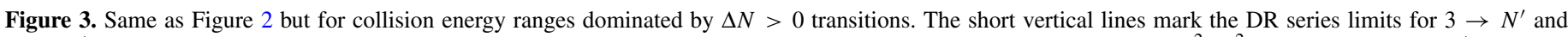

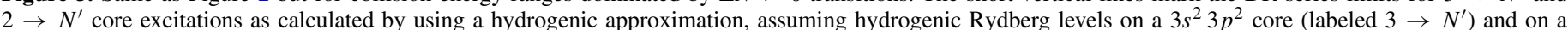

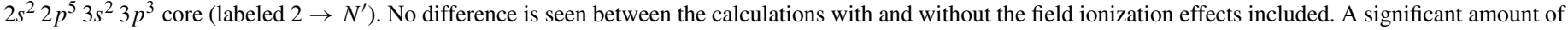
the measured DR flux is due to channels not accounted for in the theoretical calculations.

(Ralchenko et al. 2011). The next range from $\sim 330$ to $855 \mathrm{eV}$ is dominated by DR via $2 \rightarrow N$ core excitations where $N^{\prime} \geqslant 3$. We attribute the resonances seen in this range mainly to $2 \rightarrow 3$ excitations and calculate a $\kappa$ of 0.28 . For the range $\sim 855-1073 \mathrm{eV}$, no theoretical data exist and we are unable to determine $\kappa$. The $2 \rightarrow N^{\prime}$ channels cease to contribute to DR once ionization from the $N=2$ level becomes possible at $1073 \mathrm{eV}$ (Kaastra \& Mewe 1993), as is readily visible in the measured data. It is worth noting, too, that a significant amount of the measured DR flux above $\sim 60 \mathrm{eV}$ is not accounted for in the theoretical calculations.

We have also measured the MBRRC using the Target as the probe beam and the Cooler for cooling. These results are shown in Figure 4 along with the data collected using the Cooler. The differences seen below $0.001 \mathrm{eV}$ are attributed to enhancements in the RR signal as we discuss in Section 5. At higher energies, the greater resolution of the Target compared to the Cooler allows additional resonance features to be resolved, particularly for collision energies between $0.001 \mathrm{eV}$ and $0.02 \mathrm{eV}$. Note also how the Target data drop to the level of the RR background around $0.03 \mathrm{eV}$. In Section 5, these Target results are used to determine the contribution of the low energy DR resonances to the PRRC.

\section{PLASMA DR RATE COEFFICIENTS}

The derivation of the PRRC from the experimental MBRRC data has been discussed in detail in Schippers et al. (2001, 2004), Schmidt et al. (2008), and Lestinsky et al. (2009). Four points need to be considered. First, at sufficiently high collision energies, the required cross-section can be extracted by dividing the MBRRC data by the classical relative velocity $v_{\mathrm{r}}=\sqrt{2 \hat{E} / m_{\mathrm{e}}}$. For this we used the Cooler data shown in Figure 2.
Second, we need to account for recombination near various series limits into those levels which are expected to be field ionized in our experimental arrangement. Here, we took the difference between the AUTOSTRUCTURE calculations with and without field ionization effects, scaled the difference by the $\kappa$ factor for the energy range just below that where field ionization is an issue, and added the results to our measured MBRRC. The cross-section was then extracted as described above.

Third, at lower energies (here $\lesssim 0.11 \mathrm{eV}$ ), the experimental energy spread becomes comparable to $\hat{E}$. Our approximation for the cross-section breaks down and one must fit the data to extract resonance energies and strengths for the many unresolved resonances in the data. Here, we used data collected with the Target as shown in Figure 4. The Target provides much higher resolution data for extracting resonance strengths compared to the Cooler data. The fitting procedure was described in detail by Schippers et al. (2004), Schmidt et al. (2008), and Lestinsky et al. (2009).

Last, at near zero energy, the RR signal may be experimentally enhanced (Gwinner et al. 2000; Wolf \& Gwinner 2003; Hörndl et al. 2006). There may also be unresolved DR resonances. Previous work has shown that experimental enhancement of the RR MBRRC amounts to a factor of $\sim 1.5-3$ as compared to that predicted by RR theory. Any remaining difference is attributed to unresolved DR resonances. As shown in Figure 4 , the differences seen here at $\sim 10^{-4} \mathrm{eV}$ are a factor of $\sim 360$ for the Cooler and $\sim 420$ for the Target. These factors strongly suggest the presence of unresolved low energy DR resonances. Also the fact that the slopes of the Target and Cooler DR data at $\sim 10^{-3} \mathrm{eV}$ differ significantly from those predicted by RR theory supports this hypothesis. We account for this likely DR when fitting our MBRRC data by including resonances at energies of 0.08 and $0.7 \mathrm{meV}$. We calculated the PRRC with and without these resonances. The average 


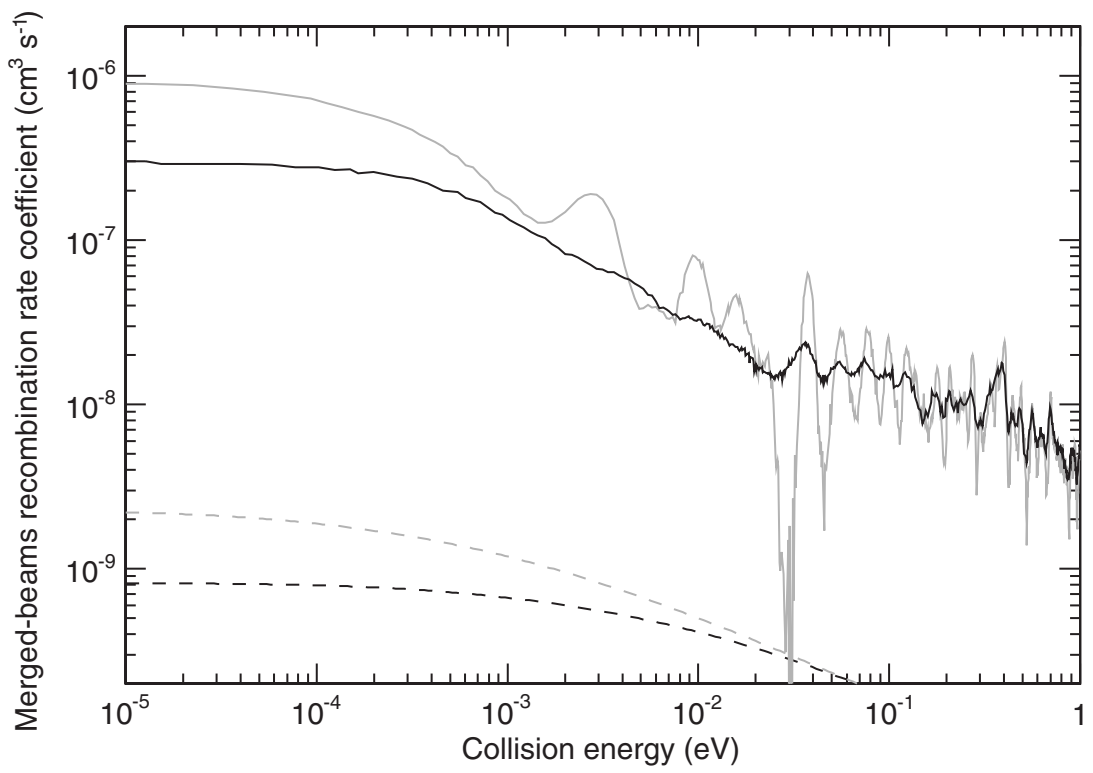

Figure 4. Comparison of the low-energy Fe XII to Fe XI MBRRC data acquired at the Cooler (black solid line, $k_{\mathrm{B}} T_{\perp}^{\mathrm{c}} \approx 13.5 \mathrm{meV}$ ) and at the Target (gray solid line, $k_{\mathrm{B}} T_{\perp}^{\mathrm{t}} \approx 1.5 \mathrm{meV}$ ). The dashed lines show the theoretical RR contribution convolved with the electron energy spreads of the Cooler (black) and Target (gray), respectively.

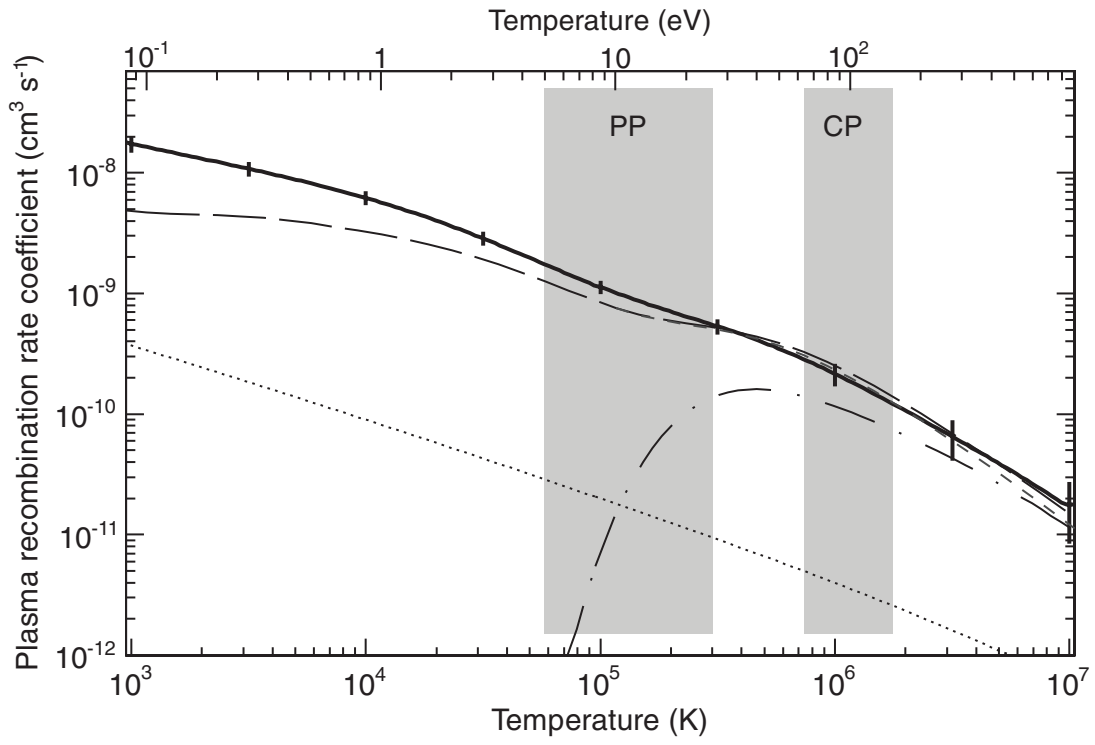

Figure 5. Comparison of the experimental and theoretical DR PRRC for Fe XII forming Fe XI. The thick solid line gives the experimental results and the error bars give the experimental uncertainty at a $1 \sigma$ confidence level. The previously recommended rate coefficient of Arnaud \& Raymond (1992) is shown by the long dash-dotted curve. The short dashed curve gives previous results of Badnell (2006b) while the long dashed curve presents our new results which extend these older calculations by including $N=3 \rightarrow 4$ and $2 \rightarrow 3$ core excitations. These two curves overlap below $\sim 3 \times 10^{5} \mathrm{~K}$. For comparison, we plot also the calculated RR PRRC (dotted line) of Badnell (2006b). The shaded areas indicate the plasma temperatures where the Fe XII abundance is $\geqslant 1 \%$ in photoionized plasmas (PPs; Kallman 2010) and in collisionally ionized plasmas (CPs; Bryans et al. 2006, 2009).

of the two PRRC results was used and half the difference between the two taken as the uncertainty. In this way, we estimate the uncertainty due to the unclear origin of these resonances. This error was then propagated quadratically into the total error budget of the PRRC. However, the contribution of these resonances to the total PRRC is insignificant above $10^{3} \mathrm{~K}$, which includes the temperatures where Fe XII is predicted to form in either photoionized or collisionally ionized gas (see Figure 5).

Taking all these points into account, we have derived the PRRC following the procedure laid out in Schippers et al. (2001, 2004), Schmidt et al. (2008), and Lestinsky et al. (2009). Figure 5 shows the results for Fe XII forming Fe XI in the temperature range of $10^{3}-10^{7} \mathrm{~K}$. The total uncertainty at an estimated $1 \sigma$ level reaches $\lesssim 15 \%$ at $10^{3} \mathrm{~K}, \sim 15 \%$ at $10^{5} \mathrm{~K}$, $\sim 20 \%$ at $10^{6} \mathrm{~K}, \sim 27 \%$ at $2 \times 10^{6} \mathrm{~K}$, and $\sim 45 \%$ at $10^{7} \mathrm{~K}$. Over the temperature range shown in the figure, the experimental DR PRRC is $\gtrsim 35$ times larger than the theoretical RR value of Badnell (2006b).

The temperature ranges where the fractional abundance of Fe XII is $\geqslant 1 \%$ of the total $\mathrm{Fe}$ abundance in photoionized plasmas (PPs) and in collisionally ionized plasmas (CPs) are indicated in Figure 5 as gray shaded areas (Bryans et al. 2006, 2009; Kallman 2010). Also plotted is the previously recommended DR rate coefficient of Arnaud \& Raymond 
Table 3

Fit Parameters $c_{i}\left(\mathrm{~cm}^{3} \mathrm{~s}^{-1} \mathrm{~K}^{3 / 2}\right)$ and $E_{i}(\mathrm{~K})$ for the Experimental DR PRRC for Fe XII Using Equation (7)

\begin{tabular}{ccc}
\hline \hline$i$ & $c_{i}$ & $E_{i}$ \\
\hline 1 & $1.38 \times 10^{-3}$ & $9.48 \times 10^{2}$ \\
3 & $5.18 \times 10^{-3}$ & $5.61 \times 10^{3}$ \\
4 & $1.33 \times 10^{-2}$ & $1.92 \times 10^{4}$ \\
2 & $2.23 \times 10^{-2}$ & $6.14 \times 10^{4}$ \\
5 & $9.52 \times 10^{-2}$ & $2.70 \times 10^{5}$ \\
6 & $2.29 \times 10^{-1}$ & $8.28 \times 10^{5}$ \\
7 & $2.94 \times 10^{-1}$ & $4.90 \times 10^{6}$ \\
\hline
\end{tabular}

(1992). These data significantly underestimate the DR PRRC at temperatures of below $2 \times 10^{5} \mathrm{~K}$, which are of particular importance for PPs. Also at temperatures relevant to CPs, their recommended DR data are up to about 2.4 times lower than our experimental results. Similar behavior has also been seen for other M-shell iron ions (Schmidt et al. 2006, 2008; Lukić et al. 2007; Lestinsky et al. 2009). The data reported by Arnaud \& Raymond (1992) represent a compilation of theoretical calculations largely from the 1970s and 1980s. We attribute the differences seen, in part, to the limitation of computer power at that time and the required approximations necessary to make the calculations tractable. More recent, state-of-the-art calculations have been performed by Badnell (2006b). However, these do not include DR via $\Delta N>0$ core excitations. We have extended those results by including DR via $2 \rightarrow 3$ and $3 \rightarrow 4$ core excitations. At PP temperatures both calculations are in significantly better agreement with experiment but still differ by up to $30 \%$ which is outside of the $1 \sigma$ experimental error bars. At CP temperatures both sets of theoretical results agree with experiment to within the experimental uncertainty, despite the significant disagreement between the MCBP theory and our experimental data on the MBRRC level (Figure 2). Obviously the averaging over the Maxwellian temperature distribution leads to a washing out of the discrepancies on the MBRRC level. Both the theoretical and experimental results indicate that $\Delta N>0$ channels contribute $\geqslant 10 \%$ to the PRRC at CP temperatures and up to $20 \%$ at $10^{7} \mathrm{~K}$.

To facilitate the use of our experimentally derived PRRC in plasma models, we have parameterized the data using the function

$$
\alpha_{\mathrm{P}}^{\mathrm{fit}}(T)=T^{-3 / 2} \sum_{i} c_{i} \exp \left(-E_{i} / T\right)
$$

The fitted parameters $c_{i}$ and $E_{i}$ are listed in Table 3. The fit accurately reproduces the experimentally derived PPRC to better than $2 \%$ over the temperature range of $10^{3}-10^{7} \mathrm{~K}$.

\section{SUMMARY}

We have measured the MBRRC for DR of Fe XII forming Fe XI over the collision energy range of $0-1500 \mathrm{eV}$. A merged electron-ion beam configuration was used at the TSR heavy-ion storage ring. Poor agreement is found between the experimental and theoretical resonance structure, particularly for energies below $\sim 35 \mathrm{eV}$. Significant differences are also found for the integrated resonance strengths over most of the measured energy range. Similar discrepancies between experiment and theory have been seen in our previous studies of DR for Fe M-shell ions (Schmidt et al. 2006, 2008; Lukić et al. 2007; Lestinsky et al. 2009).
From our experimental results, we have derived a DR PRRC for plasma temperatures of $10^{3}-10^{7} \mathrm{~K}$. This range includes the temperatures where Fe XII is predicted to be abundant in photoionized and collisionally ionized cosmic plasmas, respectively. In general, we see behavior similar to that noted for DR of other Fe M-shell ions (Schmidt et al. 2006, 2008; Lukić et al. 2007; Lestinsky et al. 2009). The previously recommended DR data of Arnaud \& Raymond (1992) underestimate the DR PRRC by orders of magnitude at temperatures relevant for PPs and by up to a factor of 2.4 for CPs. Much better agreement is found with state-of-the-art MCBP theory, though significant differences do remain at the lowest temperatures where modern theory is known to still have difficulties accurately predicting the energies of the relevant DR resonances.

We thank the accelerator and TSR group for their excellent support during the beam time. M.L., M.H., O.N., and D.W.S. were supported in part by the NASA Astrophysics Research and Analysis program and the NASA Solar and Heliospheric Physics Supporting Research program.

\section{APPENDIX A}

\section{LIFETIME BASED METHOD FOR ABSOLUTE SCALING OF MBRRC}

As discussed in Section 3.3, measurements for the lifetime of the stored ions with the Cooler off and on can be used to derive an absolute MBRRC. However, as a consequence of desorption from the surface of the vacuum chamber in the collector section, the residual gas pressure in the Cooler increases when the electron beam is turned on. Monitoring the pressure inside TSR by pressure gauges is not precise and local enough to describe such increases. Here, we explain how to account for these pressure changes without relying on direct pressure measurements.

Our measurements are performed on ground-state $\mathrm{Fe}^{11+}$ at collision energies below the threshold for EII. Essentially the only electron-driven, charge-changing reaction which can occur under these conditions is electron-ion recombination. We estimate as insignificant the contributions from electron impact excitation to a bound level followed by an ionizing collision on the residual gas in the ring. The ion beam is also free of metastables, i.e., the beam composition does not change during measurements. The only other significant processes affecting the lifetime of the stored ions are collisions with residual gas particles in the ring leading to electron capture (recombination) or loss (ionization).

The Cooler energy is kept constant during the on phase to insure that the electron collision rate coefficient to be derived is constant during measurement. Detectors downstream of the Cooler are used to monitor the various collision end products. The other electron beam device in the ring needs to be continuously on or continuously off so as not to disturb the measurement. The derivation presented here assumes that the Target is on continuously. Also, although we collected data using the Cooler, the role of the Cooler and Target may be readily interchanged.

The rate coefficient for the electron-ion recombination is given by $\alpha^{1}$. The rate coefficients for this reaction in the Cooler (c) and Target (t) are generally not identical, $\alpha_{\mathrm{c}}^{1} \neq \alpha_{\mathrm{t}}^{1}$, as each device has a different electron beam energy spread. Additionally, each device can be operated at different energies. 
Collisions of the stored ions with the residual gas in the ring can result in either electron capture from the gas (1) or ionization (2) of the ions. Both processes affect the lifetime of the stored ions. Both are pressure dependent. In the following, the associated rate coefficients for charge capture and ionization are denoted as $\beta^{1}$ and $\beta^{2}$, respectively.

We can readily write out expressions for the time $t$ dependence of the number of stored ions in the ring $N_{\mathrm{i}}$. With the Cooler off, this is given by

$$
\begin{aligned}
\frac{d N_{\mathrm{i}}^{\mathrm{off}}}{d t}= & -N_{\mathrm{i}}^{\mathrm{off}}\left[\left(\beta^{1}+\beta^{2}\right) \rho_{\mathrm{c}}^{\text {off }} \eta_{\mathrm{c}}\right. \\
& \left.+\left(\beta^{1}+\beta^{2}\right) \rho_{\mathrm{o}}^{\text {off }}\left(1-\eta_{\mathrm{c}}\right)+\alpha_{\mathrm{t}}^{1} n_{\mathrm{t}} \eta_{\mathrm{t}}\right] .
\end{aligned}
$$

Here, $\eta_{\mathrm{c}}$ and $\eta_{\mathrm{t}}$ are the fractions of the ring circumference covered by the Cooler and Target length, respectively, $n_{\mathrm{t}}$ is the Target electron density, and $\rho_{\mathrm{c}}, \rho_{\mathrm{o}}$ are the average residual gas densities in the Cooler (c) or in other sections of TSR (o). The "off" superscript is used to denote that the Cooler is off. With the Cooler on we have

$$
\begin{aligned}
\frac{d N_{\mathrm{i}}^{\text {on }}}{d t}= & -N_{\mathrm{i}}^{\text {on }}\left[\left(\beta^{1}+\beta^{2}\right) \rho_{\mathrm{c}}^{\text {on }} \eta_{\mathrm{c}}\right. \\
& \left.+\left(\beta^{1}+\beta^{2}\right) \rho_{\mathrm{o}}^{\text {on }}\left(1-\eta_{\mathrm{c}}\right)+\alpha_{\mathrm{t}}^{1} n_{\mathrm{t}} \eta_{\mathrm{t}}+\alpha_{\mathrm{c}}^{1} n_{\mathrm{c}} \eta_{\mathrm{c}}\right],
\end{aligned}
$$

where the "on" superscript signifies the Cooler is on and $n_{\mathrm{c}}$ is the Cooler electron density. The solution for these equations is of the form

$$
N_{\mathrm{i}}^{\mathrm{off} / \mathrm{on}}(t)=N_{\mathrm{i}, 0}^{\mathrm{off} / \mathrm{on}} \exp \left(-t / \tau^{\text {off } / \mathrm{on}}\right)
$$

where $N_{\mathrm{i}, 0}^{\text {off/on }}$ is the initial ion number and the ion beam lifetimes $\tau^{\text {off/on }}$ are given by

$$
\left(\tau^{\text {off }}\right)^{-1}=\left(\beta^{1}+\beta^{2}\right) \rho_{\mathrm{c}}^{\text {off }} \eta_{\mathrm{c}}+\left(\beta^{1}+\beta^{2}\right) \rho_{\mathrm{o}}^{\text {off }}\left(1-\eta_{\mathrm{c}}\right)+\alpha_{\mathrm{t}}^{1} n_{\mathrm{t}} \eta_{\mathrm{t}}
$$

$$
\begin{aligned}
\left(\tau^{\mathrm{on}}\right)^{-1}= & \left(\beta^{1}+\beta^{2}\right) \rho_{\mathrm{c}}^{\mathrm{on}} \eta_{\mathrm{c}}+\left(\beta^{1}+\beta^{2}\right) \rho_{\mathrm{o}}^{\mathrm{on}}\left(1-\eta_{\mathrm{c}}\right) \\
& +\alpha_{\mathrm{t}}^{1} n_{\mathrm{t}} \eta_{\mathrm{t}}+\alpha_{\mathrm{c}}^{1} n_{\mathrm{c}} \eta_{\mathrm{c}}
\end{aligned}
$$

We can now readily solve for $\alpha_{\mathrm{c}}^{1}$ in terms of measured quantities. With the Cooler on and off, the count rates on detectors 1 (recombination) and 2 (ionization) at a time $t$ are given by

$$
\begin{gathered}
R_{\mathrm{c}}^{1, \text { on }}(t)=N_{\mathrm{i}}^{\text {on }}(t)\left(\eta_{\mathrm{c}} \alpha_{\mathrm{c}}^{1} n_{\mathrm{c}}+\beta^{1} \rho_{\mathrm{c}}^{\text {on }} \eta_{\mathrm{c}}\right) \\
R_{\mathrm{c}}^{2, \text { on }}(t)=N_{\mathrm{i}}^{\text {on }}(t)\left(\beta^{2} \rho_{\mathrm{c}}^{\text {on }} \eta_{\mathrm{c}}\right) \\
R_{\mathrm{c}}^{1, \text { off }}(t)=N_{\mathrm{i}}^{\text {off }}(t)\left(\beta^{1} \rho_{\mathrm{c}}^{\text {off }} \eta_{\mathrm{c}}\right) \\
R_{\mathrm{c}}^{2, \text { off }}(t)=N_{\mathrm{i}}^{\text {off }}(t)\left(\beta^{2} \rho_{\mathrm{c}}^{\text {off }} \eta_{\mathrm{c}}\right) .
\end{gathered}
$$

Combining Equations (A4) and (A5) with Equations (A6)-(A9) gives

$$
\begin{aligned}
\left(\tau^{\text {on }}\right)^{-1}-\left(\tau^{\text {off }}\right)^{-1}= & \frac{R_{\mathrm{c}}^{1, \text { on }}(t)+R_{\mathrm{c}}^{2, \text { on }}(t)}{N_{\mathrm{i}}^{\text {on }}(t)}-\frac{R_{\mathrm{c}}^{1, \text { off }}(t)+R_{\mathrm{c}}^{2, \text { off }}(t)}{N_{\mathrm{i}}^{\text {off }}(t)} \\
& +\left(\beta^{1}+\beta^{2}\right)\left(\rho_{\mathrm{o}}^{\text {on }}-\rho_{\mathrm{o}}^{\text {off }}\right)\left(1-\eta_{\mathrm{c}}\right) . \quad(\text { A } 10)
\end{aligned}
$$

Direct pressure measurements do not show significant pressure changes in TSR outside of the Cooler. Therefore, we assume that the pressure in these sections is independent of the state of the Cooler beam and thus $\rho_{\mathrm{o}}^{\text {on }}=\rho_{\mathrm{o}}^{\text {off }}$. We take $t_{0}$ as the time when the Cooler is switched on or off which gives $N_{\mathrm{i}}^{\text {on }}\left(t_{0}\right)=N_{\mathrm{i}}^{\text {off }}\left(t_{0}\right) \equiv N_{\mathrm{i}}\left(t_{0}\right)$. Equation (A10) thereby simplifies to

$$
\begin{aligned}
& \left(\tau^{\text {on }}\right)^{-1}-\left(\tau^{\text {off }}\right)^{-1} \\
& \quad=\frac{R_{\mathrm{c}}^{1, \text { on }}\left(t_{0}\right)-R_{\mathrm{c}}^{1, \text { off }}\left(t_{0}\right)+R_{\mathrm{c}}^{2, \text { on }}\left(t_{0}\right)-R_{\mathrm{c}}^{2, \text { off }}\left(t_{0}\right)}{N_{\mathrm{i}}\left(t_{0}\right)} .
\end{aligned}
$$

Using Equations (A6)-(A9) to solve for $N_{\mathrm{i}}\left(t_{0}\right)$ gives

$$
N_{\mathrm{i}}\left(t_{0}\right)=\frac{R_{\mathrm{c}}^{1, \text { on }}\left(t_{0}\right)-R_{\mathrm{c}}^{1, \text { off }}\left(t_{0}\right) R_{\mathrm{c}}^{2, \text { on }}\left(t_{0}\right) / R_{\mathrm{c}}^{2, \text { off }}\left(t_{0}\right)}{\eta_{\mathrm{c}} \alpha_{\mathrm{c}}^{1} n_{\mathrm{c}}} .
$$

Combining these last two equations, we obtain

$$
\begin{aligned}
\hat{\alpha} \equiv \alpha_{\mathrm{c}}^{1}= & \frac{\left(\tau^{\text {on }}\right)^{-1}-\left(\tau^{\text {off }}\right)^{-1}}{\eta_{\mathrm{c}} n_{\mathrm{c}}} \\
& \times \frac{R_{\mathrm{c}}^{1, \text { on }}\left(t_{0}\right)-R_{\mathrm{c}}^{1, \text { off }}\left(t_{0}\right) R_{\mathrm{c}}^{2, \text { on }}\left(t_{0}\right) / R_{\mathrm{c}}^{2, \text { off }}\left(t_{0}\right)}{R_{\mathrm{c}}^{1, \text { on }}\left(t_{0}\right)-R_{\mathrm{c}}^{1, \text { off }}\left(t_{0}\right)+R_{\mathrm{c}}^{2, \text { on }}\left(t_{0}\right)-R_{\mathrm{c}}^{2, \text { off }}\left(t_{0}\right)} .
\end{aligned}
$$

The measured values used to solve Equation (A13) come from data runs such as that shown in Figure 1. The lifetimes $\tau^{\text {on }}$ and $\tau^{\text {off }}$ are obtained by fitting the decaying recombination and ionization signals with the Cooler on and off, respectively. For a given state of the Cooler, the recombination and ionization lifetimes agree to within their respective uncertainties. Here, we use the lifetime measurement from the recombination data as it has better statistics than that derived from the ionization data. The lifetime fits are extrapolated to $t_{0}$ in order to determine $R_{\mathrm{c}}^{1, \text { on }}, R_{\mathrm{c}}^{2 \text {,on }}, R_{\mathrm{c}}^{1 \text {,off }}$, and $R_{\mathrm{c}}^{2 \text {,off }}$.

The accuracy for the inferred value of $\hat{\alpha}$ as given by Equation (A13) depends on the uncertainties in the various measured quantities on the right-hand side of the equation. Here, we assume that for any variable $x$, the error $\sigma_{x}$ is uncorrelated with other variables. We took partial derivatives to calculate $\sigma_{\hat{\alpha}}$ in a linear approximation. We simplify the notation using $R_{\mathrm{c}}^{1, \text { on }}\left(t_{0}\right) \equiv R_{1, \text { on }}, \eta_{\mathrm{c}} \equiv \eta$, etc. With the aid of MATHEMATICA, and after much algebraic manipulation, we find

$$
\begin{aligned}
\sigma_{\hat{\alpha}}^{2}= & \left\{\left[\eta^{2} n^{2} R_{2, \mathrm{off}}^{2}\left(R_{1, \mathrm{off}}-R_{1, \mathrm{on}}+R_{2, \mathrm{off}}-R_{2, \mathrm{on}}\right)^{2}\right.\right. \\
& \times\left(R_{1, \mathrm{on}} R_{2, \mathrm{off}}-R_{1, \mathrm{off}} R_{2, \mathrm{on}}\right)^{2}\left(\sigma_{\tau_{\mathrm{off}}^{-1}}^{2}+\sigma_{\tau_{\mathrm{on}}^{-1}}^{2}\right) \\
& +R_{2, \mathrm{off}}^{2}\left(R_{1, \mathrm{off}}-R_{1, \mathrm{on}}+R_{2, \mathrm{off}}-R_{2, \mathrm{on}}\right)^{2} \\
& \times\left(R_{1, \mathrm{on}} R_{2, \mathrm{off}}-R_{1, \mathrm{off}} R_{2, \mathrm{on}}\right)^{2}\left(\sigma_{\eta}^{2} n^{2}+\sigma_{n}^{2} \eta^{2}\right)\left(\tau_{\mathrm{off}}^{-1}-\tau_{\mathrm{on}}^{-1}\right)^{2} \\
& +\eta^{2} n^{2} R_{2, \mathrm{off}}^{2}\left(R_{2, \mathrm{off}}-R_{2, \mathrm{on}}\right)^{2}\left(R_{1, \mathrm{on}}+R_{2, \mathrm{on}}\right)^{2} \sigma_{R_{1, \mathrm{off}}}^{2} \\
& \times\left(\tau_{\mathrm{off}}^{-1}-\tau_{\mathrm{on}}^{-1}\right)^{2}+\eta^{2} n^{2} R_{2, \mathrm{off}}^{2}\left(R_{1, \mathrm{off}}+R_{2, \mathrm{off}}\right)^{2} \\
& \times\left(R_{2, \mathrm{off}}-R_{2, \mathrm{on}}\right)^{2} \sigma_{R_{1, \mathrm{on}}}^{2}\left(\tau_{\mathrm{off}}^{-1}-\tau_{\mathrm{on}}^{-1}\right)^{2} \\
& +\eta^{2} n^{2}\left(R_{1, \mathrm{off}} R_{2, \mathrm{on}}\left(-R_{1, \mathrm{off}}-2 R_{2, \mathrm{off}}+R_{2, \mathrm{on}}\right)\right. \\
& \left.+R_{1, \mathrm{on}}\left(R_{2, \mathrm{off}}^{2}+R_{1, \mathrm{off}} R_{2, \mathrm{on}}\right)\right)^{2} \sigma_{R_{2, \mathrm{off}}}^{2}\left(\tau_{\mathrm{off}}^{-1}-\tau_{\mathrm{on}}^{-1}\right)^{2} \\
& +\eta^{2} n^{2}\left(R_{1, \mathrm{off}}-R_{1, \mathrm{on}}\right)^{2} R_{2, \mathrm{off}}^{2}\left(R_{1, \mathrm{off}}+R_{2, \mathrm{off}}\right)^{2} \sigma_{R_{2, \mathrm{on}}}^{2} \\
& \left.\times\left(\tau_{\mathrm{off}}^{-1}-\tau_{\mathrm{on}}^{-1}\right)^{2}\right] /\left[\eta ^ { 4 } n ^ { 4 } R _ { 2 , \mathrm { off } } ^ { 4 } \left(R_{1, \mathrm{off}}-R_{1, \mathrm{on}}\right.\right. \\
& \left.\left.\left.+R_{2, \mathrm{off}}-R_{2, \mathrm{on}}\right)^{4}\right]\right\} .
\end{aligned}
$$


If detector 2 is not available, additional assumptions must be made, a discussion of which is beyond the scope of this paper.

To conclude, we mention the special case described in Section 3.3 where the pressure in the Cooler does not change with switching the electron beam $\left(\rho_{\mathrm{c}}^{\text {on }}=\rho_{\mathrm{c}}^{\text {off }}\right)$ and the signal on detector 1 is dominated by electron-induced processes $\left(\alpha^{1} \gg \beta^{1}\right)$. Equation (A13) then reduces to

$$
\hat{\alpha}=\frac{\tau_{\mathrm{on}}^{-1}-\tau_{\mathrm{off}}^{-1}}{\eta n},
$$

where we have dropped the Cooler subscripts for convenience. This is equivalent to Equation (5). The associated error is given by

$$
\sigma^{2}(\hat{\alpha})=\frac{\sigma_{\tau_{\mathrm{on}}^{-1}}^{2}+\sigma_{\tau_{\mathrm{off}}^{-1}}^{2}}{\eta^{2} n^{2}}+\frac{\left(n^{2} \sigma_{\eta}^{2}+\eta^{2} \sigma_{n}^{2}\right)\left(\tau_{\mathrm{off}}^{-1}-\tau_{\mathrm{on}}^{-1}\right)^{2}}{\eta^{4} n^{4}} .
$$

\section{APPENDIX B}

\section{LIST OF ABBREVIATIONS}

CP: collisionally ionized plasma

DR: dielectronic recombination

MCBP: multi-configuration Breit-Pauli

MBRRC: merged-beam recombination rate coefficient

PP: photoionized plasma

PRRC: plasma rate coefficient

$\mathrm{RR}$ : radiative recombination

\section{REFERENCES}

Altun, Z., Yumak, A., Badnell, N. R., Loch, S. D., \& Pindzola, M. S. 2006, A\&A, 447, 1165

Altun, Z., Yumak, A., Yavuz, I., et al. 2007, A\&A, 474, 1051

Arnaud, M., \& Raymond, J. 1992, ApJ, 398, 394

Badnell, N. R. 1986, J. Phys. B, 19, 3827

Badnell, N. R. 2006a, J. Phys. B, 39, 4825

Badnell, N. R. 2006b, ApJ, 651, L73

Badnell, N. R. 2011, Comp. Phys. Commun., 182, 1528

Badnell, N. R., Foster, A., Griffin, D. C., et al. 2011, J. Phys. B, 44, 135201

Behar, E., Sako, M., \& Kahn, S. M. 2001, ApJ, 563, 497

Bryans, P., Badnell, N. R., Gorczyca, T. W., et al. 2006, ApJS, 167, 343

Bryans, P., Landi, E., \& Savin, D. W. 2009, ApJ, 691, 1540

Burgess, A. 1964, ApJ, 139, 776

Chakravorty, S., Kembhavi, A. K., Elvis, M., Ferland, G., \& Badnell, N. R. 2008, MNRAS, 384, L24

Fawcett, B. C., Kononov, E. Y., Hayes, R. W., \& Cowan, R. D. 1972, J. Phys. B, 5,1255

Grieser, M., Habs, D., von Hahn, R., et al. 1991, in IEEE Particle Accelerator Conference: Accelerator Science and Technology, ed. L. Lizana \& J. Chew (Vol. 5; New York: IEEE), 2817

Gu, M. F. 2004, ApJS, 153, 389

Gwinner, G., Hoffknecht, A., Bartsch, T., et al. 2000, Phys. Rev. Lett., 84,4822
Habs, D., Baumann, W., Berger, J., et al. 1989, Nucl. Instrum. Methods B, 43 390

Hochadel, B., Albrecht, F., Grieser, M., et al. 1994, Nucl. Instrum. Methods B, 343,401

Hoffknecht, A., Schippers, S., Müller, A., Schwalm, D., \& Wolf, A. 2001, Phys. Scr. T, 92, 402

Holczer, T., Behar, E., \& Arav, N. 2010, ApJ, 708, 981

Hörndl, M., Yoshida, S., Wolf, A., et al. 2006, Phys. Rev. A, 74, 052712

Huang, K. 1984, At. Data Nucl. Data Tables, 30, 313

Kaastra, J. S., \& Mewe, R. 1993, A\&AS, 97, 443

Kallman, T. R. 2010, Space Sci. Rev., 157, 177

Kilgus, G., Habs, D., Schwalm, D., et al. 1992, Phys. Rev. A, 49, 5730

Kraemer, S. B., Ferland, G. J., \& Gabel, J. R. 2004, ApJ, 604, 556

Lampert, A., Wolf, A., Habs, D., et al. 1996, Phys. Rev. A, 53, 1413

Lestinsky, M. 2007, High-resolution Electron Collision Spectroscopy with Multicharged Ions in Merged Beams, Dissertation, Universität Heidelberg, http://www.ub.uni-heidelberg.de/archiv/7334/

Lestinsky, M., Badnell, N. R., Bernhardt, D., et al. 2009, ApJ, 698, 648

Lestinsky, M., Badnell, N. R., Bernhardt, D., et al. 2012, ApJ, submitted

Lestinsky, M., Lindroth, E., Orlov, D. A., et al. 2008, Phys. Rev. Lett., 100, 033001

Linkemann, J., Kenntner, J., Müller, A., et al. 1995, Nucl. Instrum. Methods B, 98, 154

Lukić, D. V., Schnell, M., Savin, D. W., et al. 2007, ApJ, 664, 1244

Martinson, I., \& Gaupp, A. 1974, Phys. Rep., 15, 113

Miersch, G., Habs, D., Kenntner, J., Schwalm, D., \& Wolf, A. 1996, Nucl. Instrum. Methods A, 369, 277

Müller, A. 1999, Int. J. Mass Spectrom., 192, 9

Netzer, H. 2004, ApJ, 604, 551

Orlov, D. A., Weigel, U., Schwalm, D., Terekhov, A. S., \& Wolf, A. 2004, Nucl Instrum. Methods A, 532, 418

Pastuszka, S., Hoppe, M., Kratzmann, D., et al. 2000, J. Appl. Phys., 88, 6788

Pastuszka, S., Schramm, U., Grieser, M., et al. 1996, Nucl. Instrum. Methods A, 369, 11

Pedersen, H. B., Buhr, H., Altevogt, S., et al. 2005, Phys. Rev. A, 72, 012712

Pindzola, M. S., Griffin, D. C., \& Bottcher, C. 1986, in Atomic Process in Electron-Ion and Ion-Ion Collisions, ed. F. Brouillard (New York: Plenum), 75

Poth, H. 1990, Phys. Rep., 196, 135

Ralchenko, Y., Kramida, A., Reader, J., \& NIST ASD Team 2011, Atomic Spectra Database, Version 4.1.0 (Gaithersburg, MD: National Institute of Standards and Technology), http://physics.nist.gov/asd.

Rinn, K., Müller, A., Eichenauer, H., \& Salzborn, E. 1982, Rev. Sci. Instrum., 53,829

Sako, M., Kahn, S. M., Behar, E., et al. 2001, A\&A, 365, L168

Schippers, S. 2009, J. Phys.: Conf. Ser., 163, 012001

Schippers, S., Lestinsky, M., Müller, A., et al. 2010, Int. Rev. Atom. Mol. Phys., 1,109

Schippers, S., Müller, A., Gwinner, G., et al. 2001, ApJ, 555, 1027

Schippers, S., Schnell, M., Brandau, C., et al. 2004, A\&A, 421, 1185

Schmidt, E. W., Schippers, S., Bernhardt, D., et al. 2008, A\&A, 492, 265

Schmidt, E. W., Schippers, S., Müller, A., et al. 2006, ApJ, 641, L157

Shirai, T., Funatake, Y., Mori, K., et al. 1990, J. Phys. Chem. Ref. Data, 19, 127

Sprenger, F., Lestinsky, M., Orlov, D. A., Schwalm, D., \& Wolf, A. 2004, Nucl. Instrum. Methods A, 532, 298

Steck, M., Bisoffi, G., Blum, M., et al. 1990, Nucl. Instrum. Methods A, 287, 324

Stobbe, M. 1930, Ann. Phys., 399, 661

Träbert, E., Gwinner, G., Wolf, A., et al. 2002, J. Phys. B, 35, 671

Wissler, G. 2002, A Detector System for Charge-changing and Fragmentational Experiments on Stored Ions, Dissertation, Universität Heidelberg, http://www.ub.uni-heidelberg.de/archiv/3101

Wolf, A., Buhr, H., Grieser, M., et al. 2006, Hyperfine Interact., 172, 111

Wolf, A., \& Gwinner, G. 2003, Hyperfine Interact., 146/147, 5 